\title{
Distinguishing infinite graphs
}

\author{
Wilfried Imrich \\ Montanuniversität Leoben, A-8700 Leoben, Austria \\ wilfried.imrich@mu-leoben.at \\ Sandi Klavžar* \\ Department of Mathematics and Computer Science \\ FNM, University of Maribor \\ Gosposvetska cesta 84, 2000 Maribor, Slovenia \\ sandi.klavzar@uni-mb.si \\ Vladimir Trofimov ${ }^{\dagger}$ \\ Institute of Mathematics and Mechanics \\ Russian Academy of Sciences \\ S. Kovalevskoy 16, 620219 Ekaterinburg, Russia \\ trofimov@imm.uran.ru
}

Submitted: Dec 9, 2006; Accepted: May 2, 2007; Published: May 11, 2007

Mathematics Subject Classifications: 05C25, 05C80, 03E10

\begin{abstract}
The distinguishing number $D(G)$ of a graph $G$ is the least cardinal number $\aleph$ such that $G$ has a labeling with $\aleph$ labels that is only preserved by the trivial automorphism. We show that the distinguishing number of the countable random graph is two, that tree-like graphs with not more than continuum many vertices have distinguishing number two, and determine the distinguishing number of many classes of infinite Cartesian products. For instance, $D\left(Q_{\mathfrak{n}}\right)=2$, where $Q_{\mathfrak{n}}$ is the infinite hypercube of dimension $\mathfrak{n}$.
\end{abstract}

\section{Introduction}

The distinguishing number is a symmetry related graph invariant that was introduced by Albertson and Collins [2] and extensively studied afterward. In the last couple of

\footnotetext{
*Supported in part by the Ministry of Science of Slovenia under the grants P1-0297 and BI-AT/0708-011.

${ }^{\dagger}$ Supported in part by the Russian Foundation for Basic Research under the grant 06-01-00378. The work was done in part during the visit of Montanuniversität Leoben, Leoben, Austria in May, 2006.
} 
years an amazing number of papers have been written on the topic. Let us mention just those that are directly related to our paper: It was proved independently in [6] and [12] that for a finite connected graph $G, D(G) \leq \Delta+1$, where $\Delta$ is the largest degree of $G$, with equality if and only if $G$ is a complete graph, a regular complete bipartite graph, or $C_{5}$. For the computation of the distinguishing numbers of finite trees see [5]. Also, the distinguishing number of Cartesian products of finite graphs has been widely studied; see $[1,3,8,10,11,13]$.

In this paper we extend the study of the distinguishing number to infinite graphs. The starting point is the observation in [10] that the distinguishing number of the Cartesian product of two countable complete graphs is 2 . The proof is surprisingly simple, just as one can easily show that the distinguishing number of the hypercube of countable dimension is 2 (cf. the first part of the proof of Theorem 5.4).

It turns out that many of the results for finite graphs can easily be generalized to countably infinite ones and that, with some additional effort, one can consider graphs with cardinality $\mathfrak{c}$, that is, the cardinality of the continuum. For higher cardinalities things are more complicated and are mainly treated in the last section. We wish to add that although we use "naive" set theory, it can be easily seen that our proofs remain in force in ZFC set theory.

In this paper we prove the following results for infinite graphs $G$ : We prove that the distinguishing number of the (countable) random graph is 2 , and prove that tree-like graphs (comprising all trees without pendant vertices and many other graphs) on at most $\mathfrak{c}$ vertices have distinguishing number at most 2. For higher cardinalities we treat products of complete graphs and show that $D\left(Q_{\mathfrak{n}}\right)=2$ where $Q_{\mathfrak{n}}$ is the hypercube of arbitrary infinite dimension.

\section{Preliminaries}

Let $G$ be a graph and $X$ a set. Then an $X$-labeling of $G$ is just a mapping $V(G) \rightarrow X$. In most cases $X$ will be a set of numbers, but we will also use the set \{black, white $\}$ to label graphs. When $X$ will be clear from the context we will simply speak of labelings of $G$. Let $g$ be an automorphism of $G$ and $l$ a labeling of $G$. Then we say that $l$ is preserved by $g$ if $l(v)=l(g(v))$ for any $v \in V(G)$. A labeling $l$ of a graph $G$ is distinguishing if $l$ is only preserved by the trivial automorphism of $G$. The distinguishing number of a graph $G$ is the least cardinal number $\aleph$ such that there exists a distinguishing $X$-labeling of $G$ with $|X|=\aleph$. The distinguishing number of a graph $G$ is denoted by $D(G)$. An $X$-labeling $l_{1}$ of a graph $G_{1}$ and an $X$-labeling $l_{2}$ of a graph $G_{2}$ are isomorphic if there exists an isomorphism $\varphi: V\left(G_{1}\right) \rightarrow V\left(G_{2}\right)$ of $G_{1}$ and $G_{2}$ such that $l_{1}(v)=l_{2}(\varphi(v))$ for any $v \in V\left(G_{1}\right)$ (in this case we also say that $X$-labeled graphs $G_{1}$ and $G_{2}$ are isomorphic).

As usual, $\aleph_{0}$ stands for the cardinality of a countable set and $\mathfrak{c}$ or $2^{\aleph_{0}}$ for the cardinality of reals. We will use $\mathfrak{n}$ to denote an arbitrary finite or infinite cardinal.

The Cartesian product $G \square H$ of two finite or infinite graphs $G$ and $H$ is a graph with the vertex set $V(G) \times V(H)$, the vertices $(g, h)$ and $\left(g^{\prime}, h^{\prime}\right)$ being adjacent if $g=g^{\prime}$ and $h h^{\prime} \in E(H)$, or $h=h^{\prime}$ and $g g^{\prime} \in E(G)$. Let $g$ be a vertex of $G$. Then the set of vertices 
$\{(g, h) \mid h \in V(H)\}$ induces a subgraph of $G \square H$ isomorphic to $H$. It is called an $H$-fiber and denoted ${ }^{g} H$. Analogously, for $h \in V(H)$, the $G$-fiber $G^{h}$ is the subgraph of $G \square H$ induced by $\{(g, h) \mid g \in V(H)\}$.

Let $G_{\iota}, \iota \in I$, be an arbitrary family of graphs. Then the Cartesian product $\square_{\iota \in I} G_{\iota}$ is defined on the set of all functions $x: \iota \mapsto x_{\iota}, x_{\iota} \in G_{\iota}$, where two vertices (functions) are adjacent if there exists an index $\kappa \in I$ such that $x_{\kappa} y_{\kappa} \in E\left(G_{\kappa}\right)$ and $x_{\iota}=y_{\iota}$ for all $\iota \neq \kappa$.

We wish to point out that the Cartesian product of infinitely many nontrivial graphs is disconnected. Therefore, in this case one considers connected components of the Cartesian product and calls them weak Cartesian products. If all factors have transitive group any two components are isomorphic. Since we are only interested in connected graphs, for us a Cartesian product graph will always mean a weak Cartesian product graph.

For instance, the infinite hypercube $Q_{\mathfrak{n}}$ of dimension $\mathfrak{n}$ is defined as the Cartesian product of $\mathfrak{n}$ copies of $K_{2}$. Its vertex set is the set of all functions of a set $S$ of cardinality $\mathfrak{n}$ into $\{0,1\}$, where the preimage of 1 is finite, and where two vertices $f, g$ are adjacent if there exists one and only one element $x$ of $S$ such that $f(x) \neq g(x)$. Clearly $Q_{\mathfrak{n}}$ is idempotent, that is $Q_{\mathfrak{n}} \cong Q_{\mathfrak{n}} \square Q_{\mathfrak{n}}$.

Let $x$ be a vertex of a graph $G$. Then the neighborhood of $x$ in $G$ will be denoted $N_{G}(x)$ or $N(x)$ if $G$ is clear from the context. In addition, $B_{x}(d)$ is the set of vertices of $G$ at distance $\leq d$ from $x$.

By a tree-like graph we mean a connected graph $G$ that contains a vertex $x$ with the following property: for any vertex $y$ there exists an up-vertex $z$ with respect to $x$ (that is, a vertex with $d(x, z)=d(x, y)+1)$, such that $y$ is the only vertex from $N(z)$ that lies in an $x, z$-geodesic. In other words, for any vertex $y$ there exists a vertex $z$ such that $\{y\}=N(z) \cap B_{x}(d(x, z)-1)$.

For terms not defined here, in particular for the Cartesian product of graphs and its properties, we refer to [9].

We have already mentioned that for a finite connected graph $G, D(G) \leq \Delta+1$, where $\Delta$ is the largest degree of $G$. We conclude the section with the following analogue for infinite graphs.

Theorem 2.1 Let $\mathfrak{n}$ be a cardinal number, and $G$ a connected, infinite graph such that the degree of any vertex of $G$ is not greater than $\mathfrak{n}$. Then $D(G) \leq \mathfrak{n}$.

Proof. Suppose first that $\mathfrak{n}$ is a finite cardinal. Then $G$ is an infinite graph of bounded, finite degree and $G$ contains a one-way infinite isometric path $R$. Let $u_{0}$ be the first vertex of $R$, and label all vertices of $R$ with $\Delta$, where $\Delta$ is the largest degree of $G$. No other vertex will receive the label $\Delta$. Since $u_{0}$ is labeled $\Delta$ and adjacent to exactly one vertex labeled $\Delta$, while any other vertex of $R$ is adjacent to two vertices labeled $\Delta, u_{0}$ is fixed by every automorphism of $G$. Consequently, $R$ is fixed pointwise by every automorphism. Construct a BFS-tree of $G$ with root $u_{0}$ and label the vertices of $G$ in BFS order as follows: Let $x$ be a vertex in this order, then label its neighbors that are not yet labeled with different labels from $\{1,2, \ldots, \Delta-1\}$. The BFS order implies that the labeling is well-defined and that every newly labeled vertex is fixed by every automorphism. 
Assume next that the degree of $G$ is not bounded. The vertex set of $G$ is the union of the vertices in the BFS-levels of $G$. Since the number of vertices in every such level is at most $\mathfrak{n}$ we have that $|G| \leq \mathfrak{n}$.

We note that an infinite locally finite graph can have infinite distinguishing number. For a simple example consider the graph that is obtained from the one-way infinite path $v_{0}, v_{1}, v_{2}, \ldots$ by attaching $n$ pendant vertices to $v_{n}$ for any $n \geq 1$.

\section{Distinguishing the random graph}

In this section we determine the distinguishing number of the countable random graph. So, let $R$ be the countable random graph defined in [7]. Then (see, for example, Propositions $4.1,5.1,8.1$ and 3.1 in [4]) $R$ contains any countable graph as an induced subgraph, the automorphism group $\operatorname{Aut}(R)$ of $R$ is a vertex-transitive group of order $2^{\aleph_{0}}$, and $R$ has the following property:

(*) For any finite disjoint subsets $X$ and $Y$ of $V(R)$, there are infinitely many vertices $z$ of $R$ such that $z x \in E(R)$ for all $x \in X$ and $z y \notin E(R)$ for all $y \in Y$.

In particular, $R$ is a connected graph of diameter 2 and of countable degree.

Theorem 3.1 Let $R$ be the countable random graph. Then $D(R)=2$.

Proof. Since $\operatorname{Aut}(R) \neq 1$, we have $D(R)>1$. Let $u$ be a vertex of $R$, and let $N(u)=$ $\left\{v_{1}, v_{2}, \ldots\right\}$ be the set of neighbors of $u$ in $R$. Fix a sequence $n_{1}, n_{2}, \ldots$ of positive integers such that $n_{i}>n_{1}+\cdots+n_{i-1}$ for all $i>1$. Define a labeling $l: V(R) \rightarrow\{0,1\}$ in the following way.

Put $l(u)=0$ and $l\left(v_{i}\right)=0$ for all $i \geq 1$. By $(*)$, there exist (distinct) vertices $w_{1,1}, \ldots, w_{1, n_{1}}$ in $N\left(v_{1}\right) \backslash(\{u\} \cup N(u))$. Put $l\left(w_{1, j}\right)=0$ for all $j, 1 \leq j \leq n_{1}$, and $l(w)=1$ for all other vertices $w$ from $N\left(v_{1}\right) \backslash(\{u\} \cup N(u))$. Suppose now that, for some positive integer $k, l$ is defined on $\cup_{i=1}^{k}\left(N\left(v_{i}\right) \backslash(\{u\} \cup N(u))\right)$ in such a way that, for each $i \leq k$, there are exactly $n_{i}$ vertices in $N\left(v_{i}\right) \backslash(\{u\} \cup N(u))$ labeled 0 . Then the number $n$ of vertices in $\left(N\left(v_{k+1}\right) \backslash(\{u\} \cup N(u))\right) \cap\left(\cup_{i=1}^{k}\left(N\left(v_{i}\right) \backslash(\{u\} \cup N(u))\right)\right)$ labeled 0 (by assumption, $l$ is defined on $\left.\cup_{i=1}^{k}\left(N\left(v_{i}\right) \backslash(\{u\} \cup N(u))\right)\right)$ is $\leq n_{1}+\ldots+n_{k}<n_{k+1}$. By $(*)$, there exist (distinct) vertices $w_{k+1,1}, \ldots, w_{k+1, n_{k+1}-n}$ in $N\left(v_{k+1}\right) \backslash\left((\{u\} \cup N(u)) \cup\left(\cup_{i=1}^{k} N\left(v_{i}\right)\right)\right)$. Put $l\left(w_{k+1, j}\right)=0$ for all $j, 1 \leq j \leq n_{k+1}-n$, and $l(w)=1$ for all other vertices $w$ from $N\left(v_{k+1}\right) \backslash\left((\{u\} \cup N(u)) \cup\left(\cup_{i=1}^{k} N\left(v_{i}\right)\right)\right)$. As a result, for any positive integer $i \leq k+1, l$ is defined on $N\left(v_{i}\right) \backslash(\{u\} \cup N(u))$ and there are exactly $n_{i}$ vertices in $N\left(v_{i}\right) \backslash(\{u\} \cup N(u))$ labeled 0 . Since the diameter of $R$ is 2 , continuing in this way we get a labeling $l: V(R) \rightarrow\{0,1\}$.

By the definition of $l$, all vertices in $\{u\} \cup N(u)=\left\{u, v_{1}, v_{2}, \ldots\right\}$ are labeled 0 , and, for each positive integer $i$, the number of vertices in $N\left(v_{i}\right) \backslash(\{u\} \cup N(u))$ labeled 0 is $n_{i}$. Furthermore, $u$ is the only vertex of $R$ for which all neighbors are labeled 0 . In fact, suppose $u^{\prime}$ is another vertex of $R$ for which all neighbors are labeled 0 . By $(*)$, there exists a vertex $w$ of $R$ such that $w u^{\prime}, w v_{1}$ and $w w_{1,1}, \ldots, w w_{1, n_{1}}$ (where $w_{1,1}, \ldots, w_{1, n_{1}}$ are 
all vertices in $N\left(v_{1}\right) \backslash(\{u\} \cup N(u)$ ) labeled 0$)$ are edges of $R$, while $w u \notin E(R)$. But $w v_{1} \in E(R)$ and $w u \notin E(R)$ imply $w \in N\left(v_{1}\right) \backslash(\{u\} \cup N(u))$. At the same time, for each $j, 1 \leq j \leq n_{1}$, we have $w \neq w_{1, j}$ by $w w_{1, j} \in E(R)$. Thus $l(w)=1$ contrary to $w u^{\prime} \in E(R)$.

Let $g$ be an automorphism of $R$ preserving the labeling $l$. By the above, $g(u)=u$ and hence $g\left(v_{i}\right)=v_{i}$ for all positive integers $i$. Suppose $g(w) \neq w$ for some vertex $w$ of $R$. By $(*)$, there exists a vertex $v$ of $R$ such that $v u$ and $v w$ are edges of $R$, while $v g(w) \notin E(R)$. Since $v u \in E(R)$, we have $v=v_{i}$ for some positive integer $i$, and hence $g(v)=v$. Now $v w \in E(R)$ while $g(v) g(w)=v g(w) \notin E(R)$, a contradiction.

Thus the labeling $l$ is distinguishing.

\section{Tree-like graphs}

We now move to uncountable graphs. The main result of this section asserts that the distinguishing number of tree-like graphs of cardinality not greater than $\mathfrak{c}$ is either one or two, but the result is no longer true for larger cardinalities. We need the following somewhat technical result.

Lemma 4.1 Let $T$ be a tree with $u \in V(T)$ such that $1 \leq \operatorname{deg}(u) \leq 2^{\aleph_{0}}$ and $1<\operatorname{deg}(v) \leq$ $2^{\aleph_{0}}$ for all $v \in V(T) \backslash\{u\}$. Then there exist $2^{\aleph_{0}}$ pairwise nonisomorphic distinguishing $\{0,1\}$-labelings $l_{\alpha}^{ \pm}, \alpha \in A$ (where $\left.|A|=2^{\aleph_{0}}\right)$, of $T$ such that, for each $\alpha \in A, l_{\alpha}^{-}(u)=0$, $l_{\alpha}^{+}(u)=1$ and with respect to each of $l_{\alpha}^{-}$and $l_{\alpha}^{+}, u$ is the only vertex of $T$ with all neighbors in $T$ labeled 0 .

Proof. Let $N_{\alpha}, \alpha \in A$ where $|A|=2^{\aleph_{0}}$, be distinct subsets of the set of integers $>2$ with $\left|N_{\alpha}\right|>1$ for all $\alpha$. For each $\alpha \in A$, let $S_{\alpha}$ be the set of (infinite) sequences $\left(i_{1}, i_{2}, \ldots\right)$ such that $\left\{i_{1}, i_{2}, \ldots\right\}=N_{\alpha}$. Of course, $\left|S_{\alpha}\right|=2^{\aleph_{0}}$ for all $\alpha \in A$. For each $\alpha \in A$ and each $s=\left(i_{1}, i_{2}, \ldots\right) \in S_{\alpha}$, let $\bar{s}$ be the $\{0,1\}$-sequence $\left(\epsilon_{0}, \epsilon_{1}, \ldots\right)$ with $\epsilon_{j}=0$ if and only if $j=i_{1}, i_{1}+i_{2}, i_{1}+i_{2}+i_{3}, \ldots$ Put $\bar{S}_{\alpha}=\left\{\bar{s}: s \in S_{\alpha}\right\}$. Of course, $\left|\bar{S}_{\alpha}\right|=\left|S_{\alpha}\right|=2^{\aleph_{0}}$ for all $\alpha \in A$.

Now let $T$ be a tree with $u \in V(T)$ such that $\operatorname{deg}(v)>1$ for all $v \in V(T) \backslash\{u\}$ and $\operatorname{deg}(v) \leq 2^{\aleph_{0}}$ for all $v \in V(T)$. For each $\alpha \in A$, we define a distinguishing $\{0,1\}$-labeling $l_{\alpha}^{-}$such that $u$ and all neighbors of $u$ in $T$ are labeled 0 , and $u$ is the only vertex of $T$ with all neighbors in $T$ labeled 0 .

Since $|V(T)| \leq 2^{\aleph_{0}}$, there is an injection $v \mapsto s_{v}$ from $V(T)$ into $\bar{S}_{\alpha}$. Define $l_{\alpha}^{-}$ inductively by Steps.

As Step 1, put $l_{\alpha}^{-}(u)=0$ and $l_{\alpha}^{-}(v)=0$ for all neighbors $v$ of $u$ in $T$.

Suppose now that after Step $n, n$ a positive integer, we have a labeling of some vertices of $T$ such that

$\left(\mathrm{i}_{n}\right)$ all vertices at distance $\leq n$ from $u$ in $T$ are labeled;

$\left(\mathrm{ii}_{n}\right)$ if a vertex $v$ from $V(T) \backslash\{u\}$ is labeled and $v^{\prime} \in V(T) \backslash\{v\}$ with $d_{T}\left(u, v^{\prime}\right)+$ $d_{T}\left(v^{\prime}, v\right)=d_{T}(u, v)$ then $v^{\prime}$ and all neighbors of $v^{\prime}$ in $T$ are labeled; 
(iii ${ }_{n}$ ) all neighbors of any vertex of $T$ labeled 1 are labeled.

If not all vertices of $T$ are labeled after Step $n$ (otherwise the definition of $l_{\alpha}^{-}$is completed), Step $n+1$ is defined as follows:

Let $\left\{v_{\beta}: \beta \in B\right\}$ be the set of all vertices of $T$ which are unlabeled but have labeled neighbors (at least one; note that by ( iii $_{n}$ ) all these neighbors are labeled 0 ). For each $\beta \in$ $B$, choose an infinite path $P_{v_{\beta}}: v_{\beta, 0}=v_{\beta}, v_{\beta, 1}, \ldots$ of $T$ such that $d_{T}\left(u, v_{\beta, j}\right)-d_{T}\left(u, v_{\beta, 0}\right)=j$ for all non-negative integers $j$. By $\left(\mathrm{ii}_{n}\right)$, the vertices of $P_{v_{\beta}}$ are unlabeled. We label vertices of $P_{v_{\beta}}$ using the sequence $\bar{s}_{v_{\beta}}$. Let $X_{v_{\beta}}^{0}$ be the set of vertices $v$ of $T$ such that, for some nonnegative integer $j, v$ is a neighbor of $v_{\beta, j}$ different from $v_{\beta, j+1}, d_{T}(u, v)=d_{T}\left(u, v_{\beta, j}\right)+1$ and $v_{\beta, j}$ is labeled 0 . Analogously, let $X_{v_{\beta}}^{1}$ be the set of vertices $v$ of $T$ such that, for some non-negative integer $j, v$ is a neighbor of $v_{\beta, j}$ different from $v_{\beta, j+1}, d_{T}(u, v)=d_{T}\left(u, v_{\beta, j}\right)+1$ and $v_{\beta, j}$ is labeled 1. Let $Y_{v_{\beta}}$ be the set of vertices $v^{\prime}$ of $T$ such that $v^{\prime}$ is a neighbor of some $v \in X_{v_{\beta}}^{1}$ with $d_{T}\left(u, v^{\prime}\right)=d_{T}(u, v)+1$. Note that, by $\left(\mathrm{ii}_{n}\right)$, no vertex in $X_{v_{\beta}}^{0}, X_{v_{\beta}}^{1}$ or $Y_{v_{\beta}}$ is labeled. Put $l_{\alpha}^{-}(v)=0$ for all $v \in X_{v_{\beta}}^{0} \cup X_{v_{\beta}}^{1} \cup Y_{v_{\beta}}$. Using the same procedure for all $v_{\beta}$, where $\beta \in B$, we complete Step $n+1$. It is easy to see that after Step $n+1$ we get a partial labeling of $T$ which has properties $\left(\mathrm{i}_{n+1}\right)-\left(\mathrm{iii}_{n+1}\right)$.

We now show that the resulting labeling $l_{\alpha}^{-}: V(T) \rightarrow\{0,1\}$ is distinguishing. Since all integers in $N_{\alpha}$ are $>2$, it follows from the definition of $l_{\alpha}^{-}$that $u$ is the only vertex of $T$ with all neighbors in $T$ labeled 0 . Hence any automorphism of $T$ preserving $l_{\alpha}^{-}$stabilizes $u$. Note now that infinite paths $x_{0}, x_{1}, \ldots$ of $T$ with the following properties a) $-\mathrm{d}$ ):

a) $d_{T}\left(u, x_{j}\right)-d_{T}\left(u, x_{0}\right)=j$ for all non-negative integers $j$,

b) if $l_{\alpha}^{-}\left(x_{j}\right)=0$ for some non-negative integer $j$, then $l_{\alpha}^{-}\left(x_{j+1}\right)=1$,

c) $l_{\alpha}^{-}\left(x_{0}\right)=1$ and $d_{T}\left(u, x_{0}\right) \geq 2$,

d) if $w$ is the neighbor of $x_{0}$ with $d_{T}(u, w)=d_{T}\left(u, x_{0}\right)-1$, and $w^{\prime}$ is the neighbor of $w$ with $d_{T}\left(u, w^{\prime}\right)=d_{T}(u, w)-1$, then $l_{\alpha}^{-}(w)=l_{\alpha}^{-}\left(w^{\prime}\right)=0$,

are exactly the paths $P_{v_{\beta}}$ used in one of the Steps. Indeed, any path $P_{v_{\beta}}=x_{0}, x_{1}, \ldots$ obviously has properties a) - d). On the other hand, if an infinite path $x_{0}, x_{1}, \ldots$ of $T$ has properties a) $-\mathrm{d}$ ), then c) implies that $x_{0}$ is a vertex of some path of the form $P_{v_{\beta}}$. Furthermore, by d) we have $x_{0}=v_{\beta}$. Now a) and b) imply that $x_{0}, x_{1}, \ldots$ coincides with $P_{v_{\beta}}$.

By the definition of $l_{\alpha}^{-}$, distinct such paths have distinct labelings, thus any automorphism of $T$ preserving $l_{\alpha}^{-}$stabilizes each such path. Since for each vertex $v$ of $T$, there exists a vertex $v^{\prime}$ with $d_{T}(u, v)+d_{T}\left(v, v^{\prime}\right)=d_{T}\left(u, v^{\prime}\right)$ which is a vertex of one such path, $l_{\alpha}^{-}$is a distinguishing labeling.

Define $l_{\alpha}^{+}$by $l_{\alpha}^{+}(u)=1$ and $l_{\alpha}^{+}(v)=l_{\alpha}^{-}(v)$ for all $v \in V(T) \backslash\{u\}$. Then, with respect to $l_{\alpha}^{+}, u$ is the only vertex of $T$ with all neighbors in $T$ labeled 0 . Since $l_{\alpha}^{-}$is a distinguishing labeling, it follows that $l_{\alpha}^{+}$is a distinguishing labeling as well.

The labels of each path of the form $P_{v_{\beta}}$ from the definition of $l_{\alpha}^{-}$make it possible to reconstruct the corresponding set $N_{\alpha}$. Therefore the labelings $l_{\alpha}^{-}$of $T$ for distinct $\alpha \in A$ are (pairwise) nonisomorphic. Hence labelings $l_{\alpha}^{+}$of $T$ for distinct $\alpha \in A$ are (pairwise) nonisomorphic as well. Besides, for $\alpha, \alpha^{\prime} \in A, l_{\alpha}^{-}$and $l_{\alpha^{\prime}}^{+}$are nonisomorphic labelings of $T$, since, with respect to each of $l_{\alpha}^{-}$and $l_{\alpha}^{+}, u$ is the only vertex of $T$ with all neighbors in 
$T$ labeled 0 while $l_{\alpha}^{-}(u) \neq l_{\alpha^{\prime}}^{+}(u)$.

Theorem 4.2 Let $G$ be a tree-like graph with $\Delta \leq 2^{\aleph_{0}}$. Then $D(G) \leq 2$.

Proof. Recall that every tree-like graph $G$ contains a vertex $x$ with the following property: for any $y \in V(G)$ there exists a $z \in V(G)$ such that $\{y\}=N(z) \cap B_{x}(d(x, z)-1)$.

Let $T$ be a spanning subgraph of $G$ with the property that $x^{\prime} x^{\prime \prime} \in E(T)$ if and only if $\left\{x^{\prime}\right\}=N\left(x^{\prime \prime}\right) \cap B_{x}\left(d\left(x, x^{\prime \prime}\right)-1\right)$ or $\left\{x^{\prime \prime}\right\}=N\left(x^{\prime}\right) \cap B_{x}\left(d\left(x, x^{\prime}\right)-1\right)$.

Clearly $T$ is a forest with no finite component, and uniquely defined. Furthermore, for any connected component $C$ of $T$, there exists a unique vertex $u_{C}$ of $C$ at a smallest distance from $x$, and the valency in $T$ of any vertex of $C$, different from $u_{C}$, is $>1$. Of course $x=u_{C^{\prime}}$ for the connected component $C^{\prime}$ of $T$ containing $x$, and the set of neighbors of $x$ in $T$ coincides with the set of neighbors of $x$ in $G$.

Define a labeling $l: V(G) \rightarrow\{0,1\}$ as follows. By Lemma 4.1, there exists a distinguishing labeling $l^{-}$of $C^{\prime}$ such that $l^{-}(x)=0$ and $l^{-}(v)=0$ for all neighbors $v$ of $x$ in $T$ (or, equivalently, in $G$ ). Put $l(w)=l^{-}(w)$ for all vertices $w$ of $C^{\prime}$. Let $\left\{C_{i}: i \in I\right\}$ be the set of connected components of $T$ different from $C^{\prime}$. Then $|I| \leq 2^{\aleph_{0}}$. Hence, by Lemma 4.1, there exist distinguishing labelings $l_{i}^{+}$of $C_{i}$ for all $i \in I$ such that $l_{i}^{+}\left(u_{C_{i}}\right)=1$, $u_{C_{i}}$ is the only vertex of $C_{i}$ for which all neighbors in $C_{i}$ are $l_{i}^{+}$-labeled 0 , and, for any distinct $i^{\prime}, i^{\prime \prime} \in I$, the $l_{i^{\prime}}^{+}$labeled tree $C_{i^{\prime}}$ and the $l_{i^{\prime \prime}}^{+}$-labeled tree $C_{i^{\prime \prime}}$ are not isomorphic. For each $i \in I$ and each $w \in C_{i}$, put $l(w)=l_{i}^{+}(w)$. This completes the definition of $l$.

Let $g$ be an arbitrary automorphism of $G$ preserving $l$. By the definition of $l, x$ is the only vertex of $G$ labeled 0 for which all neighbors in $G$ are also labeled 0 . Therefore $g(x)=$ $x$ and hence $g \in \operatorname{Aut}(T)$. Since the restrictions of $l$ to distinct connected components of $T$ give pairwise nonisomorphic labeled graphs, $g$ stabilizes each connected component of $T$. As the restrictions of $l$ to any connected component $C$ of $T$ is a distinguishing labeling of $C$, we infer that $g=1$.

Thus $l$ is a distinguishing labeling.

Let $\mathfrak{n}$ be a cardinal $>2^{\aleph_{0}}$ and $T$ a tree with $x \in V(T)$ such that $\operatorname{deg}(x)=\mathfrak{n}$ and $\operatorname{deg}(y)=2$ for all $y \in V(T) \backslash\{x\}$. Of course, $D(T) \leq|V(T)|=\mathfrak{n}$. On the other side, for any set $M$ with $|M|<\mathfrak{n}$, there are $<\mathfrak{n}$ distinct sequences of elements of $M$. Thus, for any $M$-labeling $l$ of $T$, there exist two distinct infinite paths $x_{1}^{\prime}, x_{2}^{\prime}, \ldots$ and $x_{1}^{\prime \prime}, x_{2}^{\prime \prime}, \ldots$ of $T$ with $d_{T}\left(x, x_{i}^{\prime}\right)=d_{T}\left(x, x_{i}^{\prime \prime}\right)=i$ for all positive integers $i$ such that $l\left(x_{i}^{\prime}\right)=l\left(x_{i}^{\prime \prime}\right)$ for all positive integers $i$. The automorphism $g$ of $T$ defined by $g\left(x_{i}^{\prime}\right)=x_{i}^{\prime \prime}$ and $g\left(x_{i}^{\prime \prime}\right)=x_{i}^{\prime}$ for all positive integers $i$, and $g(y)=y$ for all $y \in V(T) \backslash\left\{x_{1}^{\prime}, x_{2}^{\prime}, \ldots, x_{1}^{\prime \prime}, x_{2}^{\prime \prime}, \ldots\right\}$, is a nontrivial automorphism preserving $l$. Hence $D(T)=\mathfrak{n}$. Thus the condition $\operatorname{deg}(v) \leq 2^{\aleph_{0}}$ in the hypothesis of Theorem 4.2 cannot be weakened (even for trees and even for a single vertex).

We close the section by noting that countable trees have also independently been treated by Tucker [15]. 


\section{Cartesian products of arbitrary cardinality}

In this section we generalize results about the distinguishing number of Cartesian products of complete graphs $[8,10]$ to the infinite case and find many similarities. We also determine the distinguishing number of infinite hypercubes of arbitrary dimension.

To this end we take recourse to elementary results about infinite cardinals and ordinals, transfinite induction, and the well-ordering theorem; see e.g. [14].

We also apply several basic facts about prime factorizations and automorphisms of connected infinite graphs with respect to the weak Cartesian product: Every connected, infinite graph can be uniquely represented as the weak Cartesian product of prime graphs, that is, graphs that are not the product of two nontrivial graphs; see [9]. Moreover, given a product

$$
G=\square_{\iota \in I} G_{\iota}
$$

of prime graphs, every automorphism of $G$ is induced by transpositions of isomorphic factors and automorphisms of the factors themselves. That is, if $\psi \in \operatorname{Aut}(G)$ then there exists a permutation $\pi$ of $I$ together with isomorphisms $\psi_{i}: G_{\iota} \rightarrow G_{\pi_{\iota}}$ such that

$$
(\varphi v)_{\iota}=\psi_{\pi^{-1} \iota} v_{\pi^{-1} \iota}
$$

Also, complete graphs are prime with respect to the Cartesian product.

Theorem 5.1 Let $\mathfrak{n}$ be an infinite cardinal number. Then $D\left(K_{\mathfrak{n}} \square K_{\mathfrak{n}}\right)=2$.

Proof. Let $G=G_{1} \square G_{2}$, where both $G_{1}$ and $G_{2}$ are isomorphic to $K_{\mathfrak{n}}$. Let $V=V\left(G_{1}\right)=$ $V\left(G_{2}\right)$ and $\prec$ a well-ordering of $V$ with the first element $a_{0}$.

We label every vertex $(x, y) \in G_{1} \square G_{2}$ white if $x \prec y$, otherwise we label it black.

This labelling is distinguishing. To see this we first note that every $G_{1}$-fiber has infinitely many black vertices, but that the $G_{2}$-fiber ${ }^{a_{0}} G_{2}$ has only one black vertex, namely $\left(a_{0}, a_{0}\right)$. Thus, every automorphism of $G$ is generated by automorphisms of the factors. In other words, every automorphism maps $G_{1}$-fibers into $G_{1}$-fibers and $G_{2}$-fibers into $G_{2}$-fibers.

Since $G_{1}^{a_{0}}$ is the only $G_{1}$-fiber with no white vertices it is stabilized by all automorphisms. Similarly, ${ }^{a_{0}} G_{2}$ is stabilized by all automorphisms of $G$ because it is the only $G_{2}$-fiber with only one black vertex. Thus $\left(a_{0}, a_{0}\right)=G_{1}^{a_{0}} \cap{ }^{a_{0}} G_{2}$ is fixed by all $\alpha \in \operatorname{Aut}(G)$.

If all diagonal elements $(x, x)$ are fixed, then $G_{1}^{x}$ and ${ }^{x} G_{2}$ are stabilized for every $x$. Thus $G_{1}^{x} \cap{ }^{y} G_{2}=(x, y)$ is fixed for every $(x, y) \in V(G)$ and the labeling is distinguishing.

Hence, if the labeling is not distinguishing there is a smallest ordinal $c$ in $V$ such that $(c, c)$ is not fixed by the label preserving automorphisms of $G$, but the vertices $(x, y) \in V(G)$ with $x, y \prec c$ are. Let $L$ be the union of the $G_{1^{-}}$and $G_{2}$-fibers through these vertices. Clearly $L$ is stabilized by the all label preserving automorphisms. Moreover, $G_{1}^{c}$ is the only $G_{1}$-fiber with no white vertex outside of $L$ and ${ }^{c} G_{2}$ the only $G_{2}$-fiber with just one black vertex outside of $L$. Thus $(c, c)$ is also fixed.

Theorem 5.2 Let $\mathfrak{n}$ be an infinite cardinal. Then $D\left(K_{\mathfrak{n}} \square K_{2^{\mathfrak{n}}}\right)=2$. 
Proof. We consider the complete graphs $G_{2}=K_{\mathfrak{n}}$ with vertex set $W$ and $H=K_{2^{\mathfrak{n}}}$ with vertex set $V$. Of course, $W$ is a set of cardinality $\mathfrak{n}$ and $V$ a set of cardinality $2^{\mathfrak{n}}$. Write $V$ as a disjoint union $V^{\prime} \cup V^{\prime \prime}$, where $V^{\prime}$ is a set of cardinality $\mathfrak{n}$ and $V^{\prime \prime}$ a set of cardinality $2^{\mathfrak{n}}$. Let $G_{1}$ be the subgraph of $H$ induced by $V^{\prime}$ and $G_{0}$ the subgraph of $H$ induced by $V^{\prime \prime}$. Then $\left|G_{2}\right|=\left|G_{1}\right|=\mathfrak{n}$ and $\left|G_{0}\right|=|H|=2^{\mathfrak{n}}$.

Consider the $2^{\mathfrak{n}}$ different black and white labelings of $G_{2}$ and assign them to the $G_{2^{-}}$ fibers of $G_{2} \square H$, yielding a labeling $l$ on $G_{2} \square H$. The labeling $l$ induces labelings $l^{\prime}$ of $G_{2} \square G_{1}$ and $l^{\prime \prime}$ of $G_{2} \square G_{0}$. By Theorem 5.1 we can choose $l$ such that the labeling $l^{\prime}$ is distinguishing.

If $l^{\prime \prime}$ is not distinguishing, there must be a nontrivial $l^{\prime \prime}$-preserving automorphism $\gamma$ of $G_{2} \square G_{0}$. This mapping is of the form

$$
\gamma(x, y)=(\alpha x, \beta y)
$$

where $\alpha \in \operatorname{Aut}\left(G_{2}\right)$ and $\beta \in \operatorname{Aut}\left(G_{0}\right)$. If $\alpha$ is the identity mapping $\iota$, then $\beta=\iota$ too, because any two $G_{2}$-fibers have different labelings. We can thus assume that $\alpha \neq \iota$.

We now consider the mapping

$$
\delta:(x, y) \mapsto(\alpha x, y)
$$

of $G_{2} \square H$ into itself. This mapping permutes the labelings of the $G_{2}$-fibers of $G_{2} \square H$. In other words, there is permutation $\beta^{\prime}$ of $V(H)$ such that

$$
(x, y) \mapsto\left(\alpha x, \beta^{\prime} y\right)
$$

preserves $l$. By the definition of $\gamma$ we infer that $\left.\beta^{\prime}\right|_{V\left(G_{0}\right)}=\beta$. But then $\left.\beta^{\prime}\right|_{V\left(G_{1}\right)}$ is a permutation of $V\left(G_{0}\right)$. But this is impossible, because $l^{\prime}$ is distinguishing.

If the cardinality of the second factor is bigger than $2^{\mathfrak{n}}$ the situation is strikingly different.

Proposition 5.3 Let $2^{\mathfrak{n}}<\mathfrak{m}$. Then $D\left(K_{\mathfrak{n}} \square K_{\mathfrak{m}}\right)>\mathfrak{n}$.

Proof. The number of labelings of $K_{\mathfrak{n}}$ with $\mathfrak{a} \leq \mathfrak{n}$ labels is $\mathfrak{a}^{\mathfrak{n}} \leq 2^{\mathfrak{n}}<\mathfrak{m}$.

If the generalized continuum hypothesis does not hold, there are cardinals $\mathfrak{n}$ and $\mathfrak{m}$ such that $\mathfrak{n}<\mathfrak{m}<2^{\mathfrak{n}}$. We do not know whether $D\left(K_{\mathfrak{n}} \square K_{\mathfrak{m}}\right)=2$ in this case.

Theorem 5.4 Let $\mathfrak{n}$ be an infinite cardinal. Then $D\left(Q_{\mathfrak{n}}\right)=2$.

Proof. The proof is by transfinite induction. Let $\mathfrak{n}=\aleph_{0}$. The vertices of $Q_{\aleph_{0}}$ are all 01-sequences with finitely many ones. Let $v_{i}$ be the vertex whose first $i$ coordinates are 1 , and the others 0 . We consider the one sided infinite path

$$
P=v_{0} v_{1} \ldots
$$


and label its vertices black, all the other vertices white. Clearly $P$ is fixed by every automorphism of $Q_{\aleph_{0}}$. Also, $P$ meets every $\Theta$-class of $Q_{\aleph_{0}}$ in exactly one edge. Since this edge is fixed, all $\Theta$-classes are stabilized and its edges cannot be inverted. Thus the labeling is distinguishing.

Furthermore, take all vertices with an even number of ones, throw out those that are in $N(P)$, and call this set $A$. Then $A$ is an infinite independent set of $Q_{\aleph_{0}} \backslash N(P)$. We now create $2^{\aleph_{0}}$ labelings of $Q_{\aleph_{0}}$ in which the vertices of $P$ are labeled black, the vertices of $A$ in all possible ways, and the remaining vertices white. Since no two vertices of $A$ are adjacent and no vertex of $A$ is a neighbor of a vertex in $P$, each such labeling contains exactly one black vertex (the vertex with all zeros) with exactly one black neighbor. Note also that given two copies of $Q_{\aleph_{0}}$ with distinct labelings created as above, there is no label preserving isomorphism between them.

We show next how to find a distinguishing labeling of $Q_{\aleph_{1}}$. To this end we choose $\aleph_{1}$ hypercubes $Q_{\aleph_{0}}$ and label then with distinct nonisomorphic distinguishing labelings. This is possible, because $\aleph_{1} \leq 2^{\aleph_{0}}$. Let these hypercubes, together with their labelings, be $G_{\iota}$, $\iota \in I,|I|=\aleph_{1}$. Note that every $G_{\iota}$ has exactly one black vertex that has exactly one black neighbor, we call it the root of $G_{\iota}$. We now consider the weak Cartesian product

$$
G=\square_{\iota \in I} G_{\iota}
$$

with respect to the vertex $v_{0}$ whose coordinates are the roots of the $G_{\iota}$. We also label $G$ such that every unit fiber, that is, the $G_{\iota}$-fibers through the root vertex, inherits the labeling of its factors. All other vertices are labeled white.

Clearly, $G$ is isomorphic to $Q_{\aleph_{1}}$. Also, since $v_{0}$ is the only vertex of $G$ that is labeled black and has $\aleph_{1}$ black neighbors, it is fixed by all automorphisms. Clearly this implies that the unit fibers are also fixed and the labeling is distinguishing.

Note that the subgraph induced by the black vertices in $G$ has one connected component and isolated vertices. Moreover, the black component has exactly one vertex of degree $\aleph_{1}$, all other vertices have smaller degree.

Again we can find an independent set $A$ of cardinality $\aleph_{1}$ where no vertex of $A$ is adjacent to a unit fiber vertex. This we can use to find $2^{\aleph_{1}}$ nonisomorphic distinguishing labelings of $G$. Every such labeling consists of isolated black vertices and one connected subgraph of black vertices that has a vertex, the root of $G$, of maximum degree $\aleph_{1}$.

Suppose $\mathfrak{n}$ is a cardinal with the following properties:

(i) There are $2^{\mathfrak{n}}$ nonisomorphic distinguishing labelings of $Q_{\mathfrak{n}}$.

(ii) In every one of these labelings the black vertices induce a subgraph consisting of isolated vertices and a large connected component with exactly one vertex of degree $\mathfrak{n}$ which we call the root.

Then the above arguments imply that the successor cardinal $\mathfrak{n}^{\prime}$ of $\mathfrak{n}$ also satisfies (i) and (ii).

In order to complete the proof it remains to be shown that a limit cardinal $\mathfrak{n}$ satisfies (i) and (ii) if every cardinal $\mathfrak{m}<\mathfrak{n}$ does.

For every $\mathfrak{m}$ we select a graph $G_{\mathfrak{m}} \cong Q_{\mathfrak{m}}$ together with a distinguishing labeling satisfying (i) and (ii). 
We can assume that the distinguishing labelings of the $G_{\mathfrak{m}}$ have the property that to any two $K_{2}$-factors of $G_{\mathfrak{m}}$ there is a black (isolated) vertex that projects into the vertex of the $K_{2}$-factor that is different from the root. We now consider the Cartesian product $G_{\mathfrak{m}} \square G_{\mathfrak{m}^{\prime}}, \mathfrak{m} \neq \mathfrak{m}^{\prime}$, where the unit fibers inherit the labeling from $G_{\mathfrak{m}}$ and $G_{\mathfrak{m}^{\prime}}$, and where all other vertices are white. This means that no automorphism can map a $K_{2}$-factor of $G_{\mathfrak{m}}$ into one of $G_{\mathfrak{m}^{\prime}}$. Again we consider the weak Cartesian product

$$
G=\square G_{\mathfrak{m}}
$$

where the components of the root vertex are the roots of the $G_{\mathfrak{m}}$. Again we let the unit fibers inherit the labelings of the respective factors and label everything else white. Clearly this yields a distinguishing labeling of $G$. As before we can find an independent set $A$ of cardinality $\mathfrak{n}$ that contains no vertex that is adjacent to a unit fiber. Labeling $A$ in $2^{\mathfrak{n}}$ distinct ways in black and white yields $2^{\mathfrak{n}}$ nonisomorphic distinguishing labelings of G. Clearly

$$
G=Q_{\sum_{\mathfrak{m}<\mathfrak{n}} \mathfrak{m}}=Q_{\mathfrak{n}}
$$

and satisfies (i) and (ii).

We wish to remark that this actually means that we can distinguish the Cartesian and not only the weak Cartesian product of $\mathfrak{n}$ copies of $K_{2}$ by two colors.

\section{Concluding remarks}

Very recently (Apr 4, 2007) a closely related paper [16] was published. We note that Theorem 3.1 of $[16]$ is a special case of our Theorem 4.2.

We thank a referee for carefully reading the manuscript and numerous helpful remarks.

\section{References}

[1] M. O. Albertson, Distinguishing Cartesian powers of graphs, Electron. J. Combin. 12 (2005) \#N17.

[2] M. O. Albertson and K. L. Collins, Symmetry breaking in graphs, Electron. J. Combin. 3 (1996) \#R18.

[3] B. Bogstad and L. Cowen, The distinguishing number of hypercubes, Discrete Math. 283 (2004) 29-35.

[4] P. Cameron, The random graph, pp. 333-351 in The Mathematics of Paul Erdös, II (ed. R. L. Graham and J. Nešetřil), Algorithms and Combinatorics, 14, Springer, Berlin, 1997.

[5] C. T. Cheng, On computing the distinguishing numbers of trees and forests, Electron. J. Combin. 13 (2006) \#R11. 
[6] K. L. Collins and A. N. Trenk, The distinguishing chromatic number, Electron. J. Combin. 13 (2006) \#R16.

[7] P. Erdős and A. Rényi, Asymmetric graphs, Acta Math. Acad. Sci. Hungar. 14 (1963) 295-315.

[8] M. J. Fisher, G. Isaak, Distinguishing colorings of Cartesian products of complete graphs, Discrete Math., to appear.

[9] W. Imrich and S. Klavžar, Product Graphs: Structure and Recognition, John Wiley \& Sons, New York, 2000.

[10] W. Imrich, J. Jerebic and S. Klavžar, Distinguishing Cartesian product graphs, European J. Combin., to appear.

[11] W. Imrich and S. Klavžar, Distinguishing Cartesian powers of graphs, J. Graph Theory 53 (2006) 250-260.

[12] S. Klavžar, T.-L. Wong and X. Zhu, Distinguishing labelings of group action on vector spaces and graphs, J. Algebra 303 (2006) 626-641.

[13] S. Klavžar and X. Zhu, Cartesian powers of graphs can be distinguished by two labels, European J. Combin. 28 (2007) 303-310.

[14] K. Kuratowski and A. Mostowski, Set Theory, North-Holland, Amsterdam, 1968.

[15] T. Tucker, Distinguishability of maps, preprint, 2005.

[16] M. E. Watkins and X. Zhou, Distinguishability of locally finite trees, Electron. J. Combin. 14 (2007) \#R29. 